\title{
Correlation Between HBV Infection Status, Level of Surface Antigen-antibody and Phospholipid Metabolism, Insulin Resistance
}

\author{
Li Ting ${ }^{1, ~ *, ~ C h e n ~ C h a n ~}{ }^{1,2}$, Liu Yonggang ${ }^{2}$, Yin $\operatorname{Yan}^{3}$, Li Pin ${ }^{4}$ \\ ${ }^{1}$ Digestive Endoscopy Center, First Affiliated Hospital of Jinan University, Guangzhou, China \\ ${ }^{2}$ Department of Digestive Medicine, First Affiliated Hospital of Jinan University, Guangzhou, China \\ ${ }^{3}$ Department of Infection, First Affiliated Hospital of Jinan University, Guangzhou, China \\ ${ }^{4}$ Department of Digestive Rheumatology, First Affiliated Hospital of Jinan University, Guangzhou, China
}

Email address:

540000127@qq.com (Li Ting), calin70@163.com (Chen Chan)

${ }^{*}$ Corresponding author

\section{To cite this article:}

Li Ting, Chen Chan, Liu Yonggang, Yin Yan, Li Pin. Correlation Between HBV Infection Status, Level of Surface Antigen-antibody and Phospholipid Metabolism, Insulin Resistance. American Journal of Internal Medicine. Vol. 9, No. 2, 2021, pp. 91-95. doi: 10.11648/j.ajim.20210902.17

Received: February 20, 2021; Accepted: March 6, 2021; Published: March 26, 2021

\begin{abstract}
Background: Chronic HBV infection has a continuous effect on blood sugar and blood phospholipid metabolism. Metabolic syndrome (MS) and insulin resistance (IR) are the main risk factor leading to diabetes and cardiovascular disease. At present, the relationship between chronic HBV infection and MS was still controversial. Objective: To explore the correlation between hepatitis B virus (HBV) infection status, level of surface antigen-antibody and phospholipid metabolism, insulin resistance (IR). Methods: A retrospective analysis was performed on the clinical data of 423 patients who underwent hepatitis $\mathrm{B}$ markers test in the hospital from January 2017 to June 2019. Among them, there were 95 cases with positive hepatitis B surface antigen (HBsAg) in the HBV infection positive group and 328 cases with negative HBsAg in the HBV infection negative group. The serum phospholipid fatty acid maps and IR related indexes were compared between the two groups. The correlation between the level of HBsAg antibody and phospholipid fatty acid maps, IR related indexes were analyzed by Spearman analysis. Results: The levels of saturated fatty acid (SFA) and $\mathrm{u}-6 / \mathrm{u}-3$ polyunsaturated fatty acid (PUFA) in the HBV infection positive group were higher than those in the HBV infection negative group, while n-6PUFA, n-3PUFA, PUFA, and PUFA/SFA were all lower than those in HBV infection negative group $(P<0.05)$. The levels of fasting blood glucose (FBG) and insulin resistance index (HOMA-IR) in the HBV infection positive group were higher than those in the HBV infection negative group, while fasting insulin (FINS) and islet sensitivity index (ISI) were lower than those in HBV infection negative group $(P<0.05)$. Spearman correlation analysis showed that HBeAg level was positively correlated with SFA, n-6/n-3 PUFA, FBG and HOMA-IR $(r=0.381$, $0.369,0.516,0.453, P<0.001$ ), while negatively correlated with n-3 PUFA, n-6 PUFA, PUFA, PUFA/SFA, FINS and ISI $(r=-0.322,-0.306,-0.467,-0.482,-0.465,-0.356, P<0.001)$. Conclusion: HBV infection may cause changes in the composition of serum phospholipid fatty acid and IR.
\end{abstract}

Keywords: Hepatitis B Virus, Hepatitis B Surface Antigen, Phospholipid Fatty Acid, Insulin Resistance

\section{Introduction}

There are more than 2 billion patients with hepatitis B virus (HBV) infection worldwide, of which 240 million patients with chronic hepatitis B (CHB) [1]. Although the pathogenesis of CHB has not yet been fully confirmed, the continuous replication of HBV DNA in the liver of CHB patients is the main cause of continuous liver damage [2]. The liver is the main organ for the blood lipid synthesis, transport, metabolism, and degradation in the body. Inflammatory 
factors induced by Chronic HBV infection and related antigens and antibodies will lead to chronic inflammation and immune disorders for a long time [3]. Metabolic syndrome (MS) is a pathological state in which the metabolism of the three major nutrients in the body is disordered [4], This pathological state is the main risk factor leading to diabetes and cardiovascular disease [5], and insulin resistance (IR) is the main pathogenesis of MS [6]. At present, the relationship between chronic HBV infection and MS was still controversial. The research in South Korea showed that hepatitis B surface antigen ( $\mathrm{HBsAg}$ ) positive was a protective factor for men to develop MS [7]. Research in the United States also found that the prevalence of MS in HBV-positive patients was lower than that in HBV-negative patients [8]. But a domestic retrospective cohort study found that HBV infection was a risk factor for MS [9]. Although the relationship between HBV infection and MS has not yet been determined, it has been proven that MS-related diseases could increase the risk of liver fibrosis in patients with CHB [10]. This retrospective analysis explores the correlation between hepatitis B virus (HBV) infection status, level of surface antigen-antibody and phospholipid metabolism, insulin resistance (IR), to provide a reference for clinical research.

\section{Materials and Methods}

\subsection{Object}

This study was performed on the clinical data of 423 patients in outpatient or inpatient clinics who underwent hepatitis B markers test in the hospital from January 2017 to June 2019. All patients underwent HBV-related antigen and antibody tests, and the diagnosis of CHB was based on the Viral Hepatitis Prevention and Treatment Manual [11].

Exclusion criteria: (1) Patients with hepatitis A, hepatitis C, and other liver diseases; (2) Patients who have recently received immunosuppressive agents and antiviral drugs; (3) Patients with diabetes, hypertension, obesity, and other diseases; (4) Patients with cardiovascular and cerebrovascular diseases and thyroid dysfunction; (5) Patients with incomplete clinical data.

\subsection{Methods}

\subsubsection{Patient Indicators}

Collect the patient's height, weight, waist circumference, hip circumference, blood pressure, and calculate body mass index (BMI) and waist-hip ratio (WHR). Then Fasting $12 \mathrm{~h}$ morning venous blood was collected and serum was separated. AU 2700 automatic biochemical analyzer (Japan OLYMPUS) was used to detect serum alanine aminotransferase (ALT), aspartate aminotransferase (AST), total cholesterol (TC), triglycerides (TG), high-density lipoprotein-cholesterol (HDL-C), low-density lipoprotein-cholesterol (LDL-C) level, fasting blood glucose (FBG), fasting insulin (FINS), and calculate the insulin sensitivity index (ISI) and insulin resistance index (HOMA-IR) according to the formula.

\subsubsection{HBV DNA Load and Marker Detection in Serum}

Quantitative detection of HBV-DNA load in serum was carried out by using 7900HT fluorescent quantitative PCR (American ABI). Serum HBV markers are detected by enzyme-linked immunosorbent assay (ELISA). The laboratory physicians strictly follow the instructions on the kit for detection.

\subsubsection{Detection of Serum Phospholipid Fatty Acid Maps}

Take the patient's serum after centrifugation, add a mixture containing antioxidants and an equal volume of lecithin, chloroform, and methanol to extract total lipids. And then extract the total lipids referring to the method [12] in the literature. A combined gas chromatography/mass spectrometry (GC/MS) analysis was performed with methyl esterification of phospholipids and fatty acids. Analyze the peak area of each component of free fatty acid according to GC/MS data processing software, and calculate saturated fatty acid (SFA), monounsaturated fatty acid (MUFA), polyunsaturated fatty acid (PUFA), n-3PUFA, n-6PUFA, PUFA/SFA, n-6/n-3 PUFA ratio.

\subsection{Statistical Analysis}

SPSS 20.0 statistical software was used for data analysis. Normally distributed measurement data is represented by "Mean \pm SD", and the two groups of the independent, normal distribution, and homogeneous variance data are compared using $t$ test. The count data is expressed as the number of cases or percentage, and chi-square testing is performed. Spearman analysis was used to analyze the correlation between indicators. $\mathrm{P}<0.05$ indicates that the difference is statistically significant.

\section{Results}

\subsection{Comparison of General Information of the Two Groups of Patients}

The AST, ALT, HBV DNA load logarithms and HBsAg levels of patients in the HBV infection positive group were significantly higher than those in the HBV infection negative group ( $\mathrm{P}<0.05)$, while other general data was not statistically different $(\mathrm{P}>0.05)$, Table 1 .

\subsection{Correlation Analysis of HBV Infection Status and Phospholipid Fatty Acid Maps}

Serum levels of SFA and $n-6 / n-3$ PUFA in the HBV infection positive group were higher than those in the HBV infection negative group, while n-6 PUFA, n-3 PUFA, PUFA, PUFA/SFA were lower than those in the HBV infection negative group. The difference was statistically significant $(\mathrm{P}<0.05)$, Table 2 . 


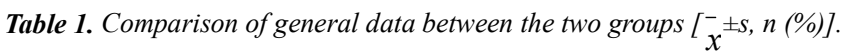

\begin{tabular}{|c|c|c|c|c|}
\hline Indicators & HBV infection positive group $(n=95)$ & HBV infection negative group $(n=328)$ & Statistics & $P$ \\
\hline Gender (male/female) & $57 / 38$ & $207 / 121$ & 0.304 & 0.582 \\
\hline Age (year) & $47.64 \pm 5.41$ & $46.83 \pm 6.32$ & 1.134 & 0.257 \\
\hline $\operatorname{BMI}\left(\mathrm{kg} / \mathrm{m}^{2}\right)$ & $25.15 \pm 2.86$ & $24.57 \pm 3.07$ & 1.646 & 0.101 \\
\hline WHR & $0.86 \pm 0.08$ & $0.85 \pm 0.06$ & 1.320 & 0.187 \\
\hline $\mathrm{SBP}(\mathrm{mmHg})$ & $138.62 \pm 12.82$ & $136.61 \pm 11.52$ & 1.459 & 0.145 \\
\hline $\mathrm{DBP}(\mathrm{mmHg})$ & $87.27 \pm 8.36$ & $86.01 \pm 7.15$ & 1.454 & 0.147 \\
\hline $\operatorname{AST}(\mathrm{U} / \mathrm{L})$ & $80.62 \pm 21.48$ & $33.65 \pm 8.67$ & 31.732 & $<0.001$ \\
\hline $\operatorname{ALT}(\mathrm{U} / \mathrm{L})$ & $56.71 \pm 13.16$ & $34.32 \pm 6.92$ & 22.063 & $<0.001$ \\
\hline $\mathrm{TC}(\mathrm{mmol} / \mathrm{L})$ & $4.91 \pm 1.16$ & $4.68 \pm 0.97$ & 1.944 & 0.053 \\
\hline $\mathrm{TG}(\mathrm{mmol} / \mathrm{L})$ & $2.62 \pm 0.78$ & $2.48 \pm 0.73$ & 1.621 & 0.106 \\
\hline HDL-C (mmol/L) & $2.84 \pm 0.85$ & $2.68 \pm 0.81$ & 1.677 & 0.094 \\
\hline LDL-C (mmol/L) & $1.35 \pm 0.34$ & $1.31 \pm 0.29$ & 1.137 & 0.256 \\
\hline HBV DNA load logarithms & $5.86 \pm 1.49$ & $1.82 \pm 0.54$ & 40.802 & $<0.001$ \\
\hline $\mathrm{HBe} A g(\mathrm{NCU} / \mathrm{mL})$ & $5.36 \pm 2.93$ & $0.32 \pm 0.11$ & 31.168 & $<0.001$ \\
\hline
\end{tabular}

Table 2. Comparison on levels of serum phospholipid fatty acid metabolic maps between the two groups $(\bar{x} \pm s)$.

\begin{tabular}{lllll}
\hline Indicators & HBV infection positive group $(\mathbf{n = 9 5})$ & HBV infection negative group $(\mathbf{n}=\mathbf{3 2 8})$ & $\boldsymbol{t}$ & \multicolumn{1}{c}{$\boldsymbol{P}$} \\
\hline SFA & $145.35 \pm 9.72$ & $138.43 \pm 7.68$ & $<.261$ & 1.220 \\
MUFA & $33.75 \pm 2.38$ & $34.06 \pm 2.12$ & 17.654 & 0.223 \\
n-3 PUFA & $88.62 \pm 3.56$ & $96.41 \pm 3.85$ & 6.707 & $<0.001$ \\
n-6 PUFA & $252.36 \pm 25.59$ & $273.36 \pm 27.23$ & 5.526 & $<0.001$ \\
PUFA & $386.25 \pm 35.51$ & $406.35 \pm 29.87$ & 3.248 & 0.001 \\
PUFA/SFA & $2.09 \pm 0.21$ & $2.20 \pm 0.31$ & 9.023 \\
n-6/n-3PUFA & $4.76 \pm 0.51$ & $4.37 \pm 0.32$ & $<0.001$ \\
\hline
\end{tabular}

\subsection{Correlation Analysis of HBV Infection Status and Insulin Resistance}

The FBG and HOMA-IR levels in the HBV infection positive group were higher than those in the HBV infection negative group, while the FINS and ISI were lower than those in the HBV infection negative group. The difference was statistically significant $(\mathrm{P}<0.05)$, Table 3 .

Table 3. Comparison on levels of IR related indexes between the two groups $(\bar{x} \pm s)$.

\begin{tabular}{llll}
\hline Indicators & HBV infection positive group (n=95) & HBV infection negative group (n=328) & $\boldsymbol{t}$ \\
\hline FPG $(\mathrm{mmol} / \mathrm{L})$ & $5.68 \pm 0.87$ & $4.59 \pm 0.64$ & 13.404 \\
FINS $(\mathrm{mIU} / \mathrm{L})$ & $13.95 \pm 5.28$ & $15.15 \pm 4.91$ & $<0.001$ \\
ISI $(\times 100)$ & $1.93 \pm 0.67$ & $2.19 \pm 0.78$ & 0.040 \\
HOMA-IR & $2.53 \pm 0.48$ & $0.67 \pm 0.23$ & 2.949 \\
\hline
\end{tabular}

\subsection{Correlation Analysis of Serum HBeAg Level and Phospholipid Metabolism}

Spearman correlation analysis showed that $\mathrm{HBeAg}$ level was positively correlated with SFA and n-6/n-3 PUFA $(\mathrm{r}=0.381,0.369, \mathrm{P}<0.001)$, while it was negatively correlated with n-3 PUFA, n-6 PUFA, PUFA, PUFA/SFA ( $\mathrm{r}=-0.322$, $-0.306,-0.467,-0.482$, all $\mathrm{P}<0.001)$, and was not correlated with MUFA $(\mathrm{P}>0.05)$, Table 4.

Table 4. Correlation between level of serum HBsAg and phospholipid metabolism.

\begin{tabular}{lll}
\hline \multirow{2}{*}{ Indicators } & HBeAg & $\boldsymbol{P}$ \\
\cline { 2 - 3 } & $\boldsymbol{r}$ & $<0.001$ \\
\hline SFA & 0.381 & 0.738 \\
MUFA & 0.035 & $<0.001$ \\
n-3 PUFA & -0.322 & $<0.001$ \\
n-6 PUFA & -0.306 & $<0.001$ \\
PUFA & -0.467 & $<0.001$ \\
PUFA/SFA & -0.482 & $<0.001$ \\
n-6/n-3PUFA & 0.369 & \\
\hline
\end{tabular}

\subsection{Correlation Analysis of Serum HBeAg Level and Insulin Resistance}

Spearman correlation analysis showed that $\mathrm{HBeAg}$ level was positively correlated with FBG and HOMA-IR ( $\mathrm{r}=0.516$, 0.453 , both $\mathrm{P}<0.001$ ), and negatively correlated with FINS and ISI ( $\mathrm{r}=-0.465,-0.356$, both $\mathrm{P}<0.001)$, Table 5 .

Table 5. Correlation between level of serum HBsAg and IR.

\begin{tabular}{lll}
\hline \multirow{2}{*}{ Indicators } & HBsAg \\
\cline { 2 - 3 } & $\boldsymbol{r}$ & $\boldsymbol{P}$ \\
\hline FBG & 0.516 & $<0.001$ \\
FINS & -0.465 & $<0.001$ \\
ISI & -0.356 & $<0.001$ \\
HOMA-IR & 0.453 & $<0.001$ \\
\hline
\end{tabular}

\section{Discussion}

China is an area with a high incidence of HBV infection, and the HBsAg seroprevalence rate is $5.49 \%$ [13]. As one of 
the main serological markers of $\mathrm{HBV}$ infection, $\mathrm{HBsAg}$ usually appears weeks before and months after the onset of symptoms, or even decades. It can be detected in the cells of patients with acute hepatitis $\mathrm{B}, \mathrm{CHB}, \mathrm{HBsAg}$ carrying, liver cirrhosis, and primary hepatocellular carcinoma. Although it could be used as the earliest evidence of HBV infection, it lacked specificity in distinguishing acute and chronic hepatitis [14]. The key to persistent liver damage in CHB patients lied in the continuous replication of the HBV DNA virus. The elevated levels of $\mathrm{HBeAg}$ in the serum may be one of the signs of active HBV replication and transmission. However, for mutant $\mathrm{HBV}$, it might not secrete $\mathrm{HBeAg}, \mathrm{HBeAg}$ could not be used to evaluate the infectivity of HBV [15]. The results showed that there was no statistical difference in blood pressure and lipid indexes between HBV positive patients and negative patients, while HBV DNA load and HBsAg level were significantly higher than those of negative patients, suggesting that HBV DNA load and HBsAg level were still important reference indicators for the diagnosis of $\mathrm{HBV}$ infection.

Studies have shown that the serum TG level of patients with chronic HBV infection was significantly lower than that of the normal control group, and apolipoprotein B100, the main component of HDL-C and LDL-C, was also significantly lower than that of the control group, suggesting that HBV infection could change blood lipid metabolism after liver damage [16]. Free fatty acids were mainly composed of oleic acid, palmitic acid, and linoleic acid, which combine with albumin and were most actively oxidized in the liver and muscles [17]. The results of the study showed that the serum SFA level of the HBV infection positive group was significantly higher than that of the HBV infection negative group. This might due to the mild inflammation caused by HBV in patients with positive infections, which would reduce the liver's $\beta$-oxidation capacity [18]. Therefore, the SFA decomposed from adipose tissue could not be synthesized and transformed in time, and the increase of SFA would cause changes in liver cell mitochondrial function and structure, which causing liver cell apoptosis [18]. The study also found that UFA was significantly reduced, among which n-6 PUFA and n-3 PUFA were the concerns of medical nutrition. Studies have shown that FINS and HOMA-IR of subjects were significantly increased after a high n-6/n-3 PUFA diet, indicating that $n-6 / n-3$ PUFA might reduce insulin sensitivity and cause insulin resistance [19]. This study found that positive HBV infection caused changes of phospholipid fatty acid maps in the body, and phospholipid fatty acids, as a biochemical indicator reflecting fatty acid metabolism in the body, were the main indicator reflecting the type of dietary fatty acids [20]. Liver damage caused by HBV infection could cause disorders of fat metabolism, increase free fatty acids in the body, and induce MS. Spearman correlation analysis showed that HBeAg level was positively correlated with SFA, n-6/n-3 PUFA, and negatively correlated with n-3 PUFA, n-6 PUFA, PUFA, PUFA/SFA, further confirming that HBV continuous replication caused liver damage, which in turn caused phospholipid fatty acid metabolism disorders.
The liver was an important organ for blood-sugar metabolism, and its functional damage could cause abnormal blood sugar rise [21]. The results of the study showed that the serum FBG level and HOMA-IR of HBV positive patients were significantly higher than those of HBV negative patients. FINS and ISI were significantly lower than those of HBV negative patients. This might because the $\mathrm{X}$ protein of the $\mathrm{HBV}$ virus could act on the liver insulin signal pathway [22]. Current studies have found that HBV did not directly cause liver damage, but the immune regulation process of the body cleared the virus. The continuous release of inflammatory cytokines could mediate the activation of multiple signaling pathways, causing the phosphorylation of INS receptor substrate -1 serine in insulin-sensitive cells such as liver cells, adipocytes, and muscle cells, blocking insulin signal transduction and mediating IR [23]. In addition to directly blocking signal transduction, elevated SFA caused by HBV infection might also promote the IR process. C16:0, as the main component of SFA, could stimulate glucose transport in the short term because it could act as an insulin analog, but it would cause IR in the long term [24]. The study also found that HBeAg levels were positively correlated with FBG and HOMA-IR, and negatively correlated with FINS and ISI, further confirming that HBV infection was related to the occurrence of type 2 diabetes. Shen et al [25] found that HBsAg positive patients had a higher risk of diabetes, and CHB course, HBV load, and liver cirrhosis were all related to type 2 diabetes.

In summary, the body's inflammatory response and specific antigen antibodies caused by chronic HBV infection would all act on the liver, leading to liver metabolic dysfunction. HBV infection could increase the level of SFA in phospholipid fatty acids, while decreases the level of UFA, which also caused IR. HBV infection, MS, and insulin resistance affected each other and promoted the development of the disease. For patients with HBV infection, in addition to clinical antiviral treatment, it was necessary to control the patient's blood sugar, phospholipid fatty acid and improve IR. However, this retrospective study had a small sample size, and the current relationship between $\mathrm{HBV}$ infection and MS was still controversial. In the future, prospective, multi-center, larger sample size studies are needed to explore the relationship between HBV infection, MS, and diabetes.

\section{Conclusion}

The body's state caused by chronic HBV infection may cause changes in the composition of serum phospholipid fatty acid and IR. HBV infection, MS, and IR affected each other and promoted the development of the disease, which could guide clinical treatment.

\section{References}

[1] Polaris Observatory Collaborators. Global prevalence, treatment, and prevention of hepatitis B virus infection in 2016: a modelling study [J]. Lancet Gastroenterol Hepatol, 2018, 3 (6): 383-403. 
[2] Indolfi G, Easterbrook P, Dusheiko G, et al. Hepatitis B virus infection in children and adolescents [J]. Lancet Gastroenterol Hepatol, 2019, 4 (6): 466-476.

[3] Chen XL, Han YD, Wang H. Relations of hepatic steatosis with liver functions, inflammations, glucolipid metabolism in chronic hepatitis B patients [J]. Eur Rev Med Pharmacol Sci, 2018, 22 (17): 5640-5646.

[4] McCracken E, Monaghan M, Sreenivasan S. Pathophysiology of the metabolic syndrome [J]. Clin Dermatol, 2018, 36 (1): $14-20$.

[5] Mendrick DL, Diehl AM, Topor LS, et al. Metabolic syndrome and associated diseases: from the bench to the clinic [J]. Toxicol Sci, 2018, 162 (1): 36-42.

[6] Gluvic Z, Zaric B, Resanovic I, et al. Link between metabolic syndrome and insulin resistance [J]. Curr Vasc Pharmacol, 2017, 15 (1): 30-39.

[7] Choi JS, Han KJ, Lee S, et al. Serum HBV surface antigen positivity is associated with low prevalence of metabolic syndrome in Korean adult men [J]. J Epidemiol, 2015, 25 (1): 74-79.

[8] Jinjuvadia R, Liangpunsakul S. Association between metabolic syndrome and its individual components with viral hepatitis B [J]. Am J Med Sci, 2014, 347 (1): 23-27.

[9] Zhou Y, Cui Y, Deng H, et al. Association between hepatitis B virus infection and metabolic syndrome: a retrospective cohort study in Shanghai, China [J]. BMC Public Health, 2014, 516 (14): 1-6.

[10] Cai S, Ou Z, Liu D, et al. Risk factors associated with liver steatosis and fibrosis in chronic hepatitis B patient with component of metabolic syndrome $[\mathrm{J}]$. United European Gastroenterol J, 2018, 6 (4): 558-566.

[11] Chinese Society of infectious and parasitic diseases and Chinese Society of Liver Diseases. Viral Hepatitis Prevention and Treatment Manual [J], CHINESE JOURNAL OF HEPATOLOGY, 2000, 8 (6): 324-328.

[12] Li Fu L, Li B, Liu Y, et al. A Comparative Study of Serum Fatty Acid in Patients with Psoriasis Vulgaris and Normal Individuals [J], The Chinese Journal of Dermatovenereology, 2008, 22 (11): 644-646.

[13] Schweitzer A, Horn J, Mikolajczyk RT, et al. Estimations of worldwide prevalence of chronic hepatitis B virus infection: a systematic review of data published between 1965 and 2013 [J]. Lancet Lond Engl, 2015, 386 (10003): 1546-1555.
[14] Kuhns MC, Holzmayer V, McNamara AL, et al. Improved detection of early acute, late acute, and occult Hepatitis B infections by an increased sensitivity HBsAg assay [J]. J Clin Virol, 2019, 118: 41-45.

[15] Chen P, Xie Q, Lu X, et al. Serum HBeAg and HBV DNA levels are not always proportional and only high levels of $\mathrm{HBeAg}$ most likely correlate with high levels of HBV DNA: A community-based study [J]. Medicine (Baltimore), 2017, 96 (33): e7766.

[16] Ming Y. Relationship between hepatitis B virus infection and metabolic syndrome $[\mathrm{J}]$, Journal of Chinese Practical Diagnosis and Therapy, 2017, 96 (33): e7766.

[17] Suiter C, Singha SK, Khalili R, et al. Free fatty acids: circulating contributors of metabolic syndrome $[\mathrm{J}]$. Cardiovasc Hematol Agents Med Chem, 2018, 16 (1): 20-34.

[18] Wu YL, Peng XE, Zhu YB, et al. Hepatitis B virus X protein induces hepatic steatosis by enhancing the expression of liver fatty acid binding protein [J]. J Virol, 2015, 90 (4): 1729-1740.

[19] Li N, Yue H, Jia M, et al. Effect of low-ratio n-6/n-3 PUFA on blood glucose: a meta-analysis [J]. Food Funct, 2019, 10 (8): 4557-4565.

[20] Hodge AM, Simpson JA, Gibson RA, et al. Plasma phospholipid fatty acid composition as a biomarker of habitual dietary fat intake in an ethnically diverse cohort [J]. Nutr Metab Cardiovasc Dis, 2007, 17 (6): 415-426.

[21] Lee JG, Lee S, Kim YJ, et al. Association of chronic viral hepatitis B with insulin resistance [J]. World J Gastroenterol, 2012, 18 (42): 6120-6126.

[22] Kim K, Kim KH, Cheong J. Hepatitis B virus X protein impairs hepatic insulin signaling through degradation of IRS1 and induction of SOCS3 [J]. PLoS One, 2010, 5 (3): e8649.

[23] Zhu H, Wang Y, Yu L, et al. Serological and molecular analysis on the relationships between type 2 diabetes mellitus and hepatitis B virus infection [J]. J Infect Dev Ctries, 2016, 10 (8): 837-844.

[24] Xiao X, Liu J, Luo X. Serum phospholipid fatty acid and insulin resistance in patients with metabolic syndrome $[\mathrm{J}]$, Chinese Journal of Disease Control and Prevention, 2012, 16 (5): 388-391.

[25] Shen Y, Zhang J, Cai H, et al. Identifying patients with chronic hepatitis $\mathrm{B}$ at high risk of type 2 diabetes mellitus: a cross-sectional study with pair-matched controls [J]. BMC Gastroenterol. 2015, 32 (15): 1-8. 